\title{
Genetic marker analysis of spatial and temporal relationships among existing populations and new infestations of the Mediterranean fruit fly (Ceratitis capitata)
}

\author{
DAVID S. HAYMER* ${ }^{*}$ MEI HE \& DONALD O. McINNIS $†$ \\ Department of Genetics and Molecular Biology, University of Hawaii, Honolulu, Hawaii 96822 and $†$ ARS, USDA, \\ Tropical Fruit and Vegetable Laboratory, Honolulu, Hawaii 96822, U.S.A.
}

\begin{abstract}
Genetic relationships among temporally and spatially defined populations of the Mediterranean fruit fly, Ceratitis capitata, have been analysed using markers produced by arbitrarily primed polymerase chain reaction (PCR). Populations sampled include various geographical localities around the world with established Mediterranean fruit fly populations and localities, such as California, where the status of the pest is controversial. Marker pattern relationships for these different populations have been used to determine genetic relatedness as well as to examine potential patterns of gene flow and movement of this pest species. Based on the extent to which markers are shared between these populations, it is clear that there is a range of genetic relationships. Samples from some regions, such as southern California and Guatemala, appear to be quite closely related to each other, whereas others, such as those from Greece and Argentina, show relatively little in common with any other sampled population. Multiple samplings from specific geographical sites within Guatemala and California have also been analysed in both spatial and temporal terms. The relationships among the infestations within California from recent years are quite different compared with similar populations sampled within Guatemala. This indicates that they are more likely to represent multiple, independent infestation events.
\end{abstract}

Keywords: arbitrary primed PCR, Ceratitis capitata, genetic markers, medfly, RAPD-PCR.

\section{Introduction}

In recent history, the Mediterranean fruit fly, Ceratitis capitata (medfly), has clearly demonstrated its ability to expand its host range rapidly and occupy new habitats around the world, especially where certain forms of intensive agriculture are practised. Beginning from a probable origin in East Africa, within the last 200 years the medfly has become established in several countries in the Mediterranean region, Central and South America, the Hawaiian islands and parts of Australia (Saul, 1986; Fimiani, 1989; Fischer-Colbrie \& Busch-Petersen, 1989). In addition, in recent years numerous sporadic infestations of this pest have occurred in the mainland U.S., in particular in California. The origin of these infesting flies has remained unclear,

*Correspondence. E-mail: dhaymer@hawaii.edu and the extent to which different infestations occurring from year to year in California are related to each other has remained controversial (Carey, 1991). We use the terms population and infestation interchangeably to emphasize the transient nature of the worldwide distribution of this pest.

Situations such as the sporadic infestations occurring in the mainland U.S. serve as a unique opportunity to monitor the genetic dynamics of an invasion process. Knowledge of the geographical origin of infesting flies can serve to identify actual or potential paths of gene flow as well as bottleneck effects accompanying such movements. In cases in which invasions have been observed to occur in consecutive years or in multiple localities (as has been the case recently in California), the temporal and spatial relationships between different infestations of these pests can provide a critical insight into the dynamics of the colonization process. An under- 
standing of this process has important implications for monitoring species movements in the broadest sense, not only for pest species, such as the medfly.

Suitably variable genetic markers can be used to establish relationships between existing populations and to document the dynamics of movement of pest species from existing areas to new habitats or geographical localities. In species such as the medfly, methods based on PCR amplification (Mullis et al., 1986) of anonymous genomic DNA sequences, using arbitrary (Welsh \& McClelland, 1990) or random (Williams et al., 1990) primers, are appropriate for identifying such markers. Although these methods may represent something of a compromise in terms of classical genetic analyses (Black, 1993; Slade et al., 1993), they clearly identify highly variable genetic markers relatively quickly and easily (Welsh et al., 1995), and they are not constrained by the extent of prior knowledge of the genetics or molecular biology of such organisms (Haymer, 1994).

These types of markers can be diagnostic of different populations, which may be acting as potential sources of origin of pest invasions (Williams $e t$ $a l ., 1994)$. They can also be used to monitor the fate of invasion attempts by documenting the nature and extent of genetic relationships among multiple infestations. Genetic data such as these can be crucial in distinguishing between competing hypotheses that have been proposed to explain the occurrence of the medfly in California (Carey, 1991). One hypothesis suggests that the various infestations are outbreaks of a widespread established population that has merely been suppressed, but not eradicated. Another hypothesis suggests that the different infestations must represent independent events, because of success in eradication each time the medfly has appeared. Resolution of this issue clearly has important implications for documenting the success of eradication efforts.

We previously used the method of arbitrary priming known as random amplified polymorphic DNA polymerase chain reaction (RAPD-PCR; Williams et al., 1990) to identify appropriate diagnostic genetic markers in sample collections from localities around the world with established medfly populations (Haymer \& McInnis, 1992). Gasperi et al. (1991), Malacrida et al. (1992) and Baruffi et al. (1995) have previously used allozymes and certain DNA markers to document the relationships among some medfly populations. Here, we extend the analysis using diagnostic genetic markers to examine the relationships of various 'established' medfly populations to each other and to recent infestations occurring in several localities within California in the years 1992-94, for the purpose of identifying potential paths of movement of this pest and potential sources of origin of the infestations. Genetic relationships of spatially and temporally defined populations from within California have also been characterized to determine which of the alternative scenarios of single vs. multiple introductions appears to be more likely.

\section{Materials and methods}

\section{Fly specimens}

Individual medfly specimens were obtained from wild collections, the Medfly Germplasm Repository (APHIS, USDA) and the California Department of Food and Agriculture. Because of import restrictions, in most cases only dead specimen material could be obtained. Total genomic DNA was extracted according to the method described in Haymer \& McInnis (1992) using specimens that had been freshly killed and either frozen at $-80^{\circ} \mathrm{C}$ or stored in ethanol (minimum of 70 per cent) until use. Extracted material from each specimen was checked on a 'yield' gel for quantity and quality, and an appropriate dilution was made to allow approximately $10 \mathrm{ng}$ of template to be used in each PCR reaction.

\section{PCR reactions and quantitation of results}

PCR reactions were set up using AmpliTaq DNA polymerase and conditions described by Perkin Elmer. PCR reactions were conducted on a Coy TempCycler II in $100 \mu \mathrm{L}$ volumes using $10 \mathrm{ng}$ of template DNA and $40 \mathrm{pmol}$ of a typical primer (obtained from Operon). The cycling parameters were similar to those described by Williams et al. (1990), except that we included an initial denaturation step of $2 \mathrm{~min}$ at $94^{\circ} \mathrm{C}$ (one cycle) and limited the total number of repeating cycles to 40 (in order to cut down on spurious weak bands). The cycling profile consisted of a $1-\mathrm{min}$ denaturation at $94^{\circ} \mathrm{C}$, a 1 -min annealing at $36^{\circ} \mathrm{C}$ and $2 \mathrm{~min}$ for extension at $72^{\circ} \mathrm{C}$. Amplification products were visualized using ethidium bromide staining of DNA after electrophoresis in 1.2 per cent agarose gels and polaroid photography under ultraviolet light. Gel pictures were scanned into a computer and bands were matched using the Gel Reader program available from the NCSA. Bands of weak intensity were checked for reproducibility and were not included in the analysis if considered to be unreliable. For each primer, a numerical designation was assigned to each band produced by each individual. A series of 
diagnostic gels was run to compare representative individuals from each population side by side to confirm band similarities or differences. Co-migrating bands in different individuals were assumed to represent identical allelic types. A total of 139 bands were scored in this analysis.

After scoring each band in each individual, a similarity index devised by Nei \& Li (1979) was used to compare the band patterns of individuals within as well as between populations. This index reflects the extent of band sharing calculated as $2 N_{a b} /\left(N_{a}+N_{b}\right)$, where $N_{a b}$ is the number of bands common to individuals $a$ and $b . N_{a}$ and $N_{b}$ are the total number of bands in individuals $a$ and $b$, respectively. The value produced by this index ranges from 0 , representing no band sharing, to 1 representing complete identity. The within- or between-population values are based on pairwise comparisons between individuals for a particular primer. Values obtained were then averaged over primers.

The between-population similarity indices were also converted to distance values using the relationship $d=1-s$ (Swofford \& Olsen, 1990; Khambampati et al., 1992). These between-population distance values were used as the input matrix for a neighbour-joining tree (Saitou \& Nei, 1987) to depict population relationships graphically. Trees were constructed using PHYLIP version 3.4 available from J. Felsenstein. Band representations were also converted to frequency data for each population, and these were used to calculate a distance measure (Nei, 1975) as input for another neighbour-joining tree, which could be subjected to bootstrap analysis.
The bootstrap values shown in Fig. 5 were obtained using the 'gene frequencies' option within the program SEQBOOT, version 3.5 (also available from $\mathrm{J}$. Felsenstein).

Student's $t$-test was employed as a statistical measure to compare mean population similarity values after determining that the criterion for homogeneity of variances was met.

\section{Results}

After screening more than 80 arbitrary primers, we identified a set of six considered to be informative for the purpose of resolving genetic marker differences between these populations. The sequences of the informative primers are as follows: (M12) 5'-GGGACGTTGG-3'; (M14) 5'-AGGGTCGTTC3'; (P14) 5'-CCAGCCGAAC-3'; (X16) 5'-CTCTGTTCGG-3'; (X18) 5'-GACTAGGTGG-3'. We initially identified these primers as informative by testing them on a minimum of five individuals from several wild collections used as reference populations. These reference populations represent countries or regions of the world where the medfly is established, including Hawaii, Guatemala, Greece and Argentina. The informative primers were then used to analyse a number of specimens from all the populations available (see Table 1), including individuals representing Californian infestations from the years 1992, 1993 and 1994 (includes northern and southern California), both to make direct comparisons with each of the reference populations from around the world and to consider the relation-

Table 1 Numbers of medfly analysed from different populations*

\begin{tabular}{lllc}
\hline Region & Specific locality & Year & No. of individuals analysed \\
\hline Hawaii & Mauna Loa & 1992 & 17 \\
Hawaii & Kauai & 1993 & 20 \\
Guatemala & Mundo Nuevo & 1994 & 19 \\
Guatemala & Finca Mujulia & $1994 \mathrm{a}$ & 20 \\
Guatemala & Finca Mujulia & $1994 \mathrm{~b}$ & 20 \\
Guatemala & Finca Mujulia & 1995 & 20 \\
Greece & Chios & 1993 & 10 \\
Argentina & Concordia & 1993 & 10 \\
California & Northern (San Jose) & 1992 & 9 \\
California & Southern (LA area) & 1992 & 24 \\
California & Southern (LA area) & 1993 & 12 \\
California & Southern (Ventura) & 1994 & 12 \\
\hline
\end{tabular}

*Finca Mujulia 1994a and 1994b were collected in February and April, 1994, respectively. LA area 1992 includes Pasadena, 1993 includes Granada Hills and various Los Angeles addresses. 
ships of the different Californian infestations to each other.

Figures 1 and 2 show examples of gels produced comparing representative individuals from each of several populations for two of the informative primers. Each lane contains the RAPD-PCR products produced for individuals from a number of different populations. In both figures, an arrow indicates an example of a band shared by individuals from two different regions, for example Guatemala and California.

Genetic relationships within and between all these populations were quantified using the similarity index to determine the extent of band sharing seen in comparisons of individuals both within and between populations. The results of these similarity index calculations can be seen in Table 2. Withinpopulation similarity values are presented along the diagonal of the matrix - all other numbers are between-population comparisons. The within-population values are all greater than 0.5 , consistent with the fact that most individuals within a population share similarities in their genetic makeup. The between-population comparisons vary over a much broader range, reflecting the fact that populations may be genetically similar or different as a result of their evolutionary histories.

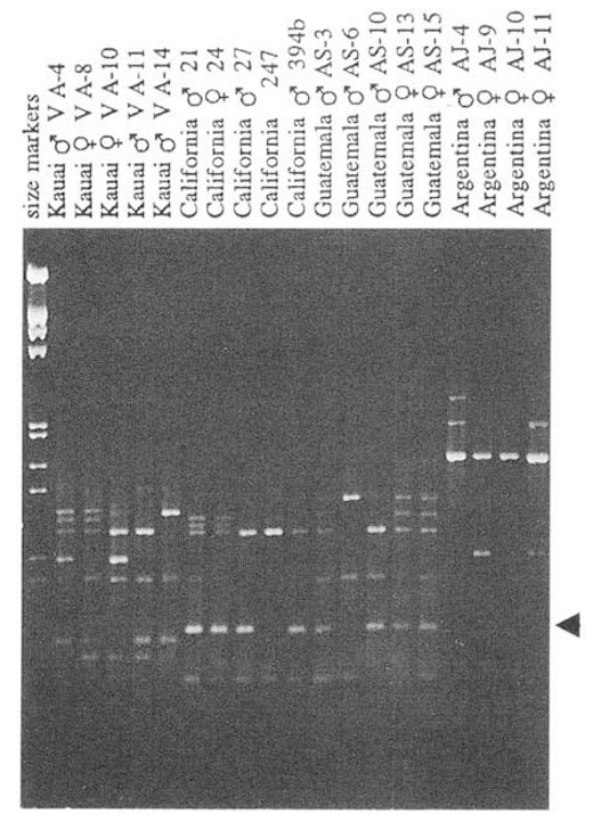

Fig. 1 Comparison of genetic marker patterns produced in representative individual medflies from Hawaii (Kauai, 93), California (LA area, 92), Guatemala (Finca Mujulia, 94a) and Argentina (Concordia, 93) using primer P14. An arrow indicates an example of a band shared only between flies from Guatemala and California (see text).

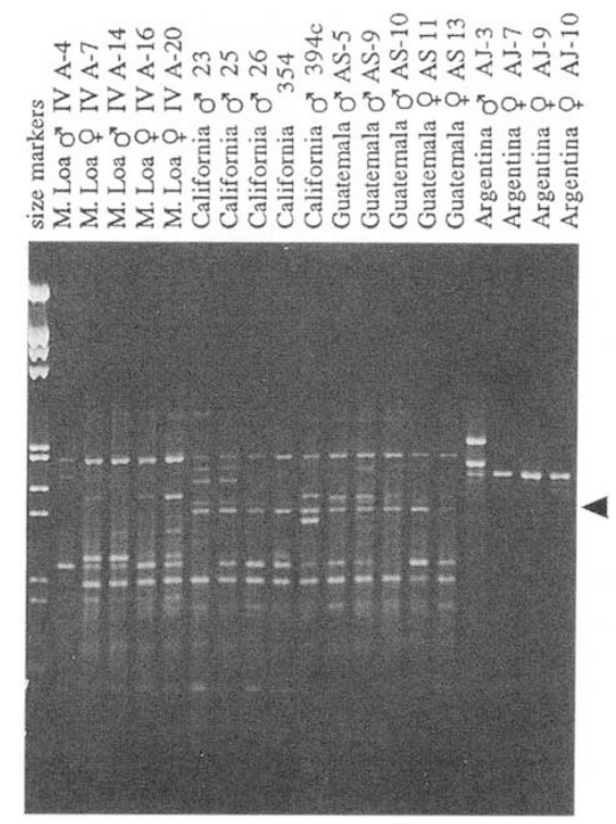

Fig. 2 Comparison of genetic marker patterns produced in representative individual medflies from Hawaii (M. Loa, 92), California (LA area, 92), Guatemala (Finca Mujulia, 94a) and Argentina (Concordia, 93) using primer M14. An arrow indicates an example of a band shared only between flies from Guatemala and California (see text).

A neighbour-joining tree (Fig. 3), constructed using a distance matrix derived from the similarity index, graphically depicts these relationships. Samples representing subpopulations from the same region exhibit the closest relationships, for example the two Hawaiian samples (M. Loa and Kauai) which cluster together. The same is true for the different spatially or temporally defined Guatemalan samples. However, the different Californian samples do not cluster closely with each other. The most extreme example of this can be seen for the sample from northern California (San Jose 92), which separates completely from any of the southern California samples.

To analyse further the relationships of the different Californian infestations to each other, the similarity values obtained here were also directly compared with similar subpopulations sampled from Guatemala. Figure 4 compares the averages of the within- and between-subpopulation similarities for these two areas. For the Guatemalan samples, the between-subpopulation similarity values are relatively high, exhibit relatively little variation $\left(s^{2}=0.0027\right)$ and are close to the average of the within-subpopulation values. For the Californian samples, the between-subpopulation values are 
Table 2 Within- and between-population similarity values for tested populations of medfly*

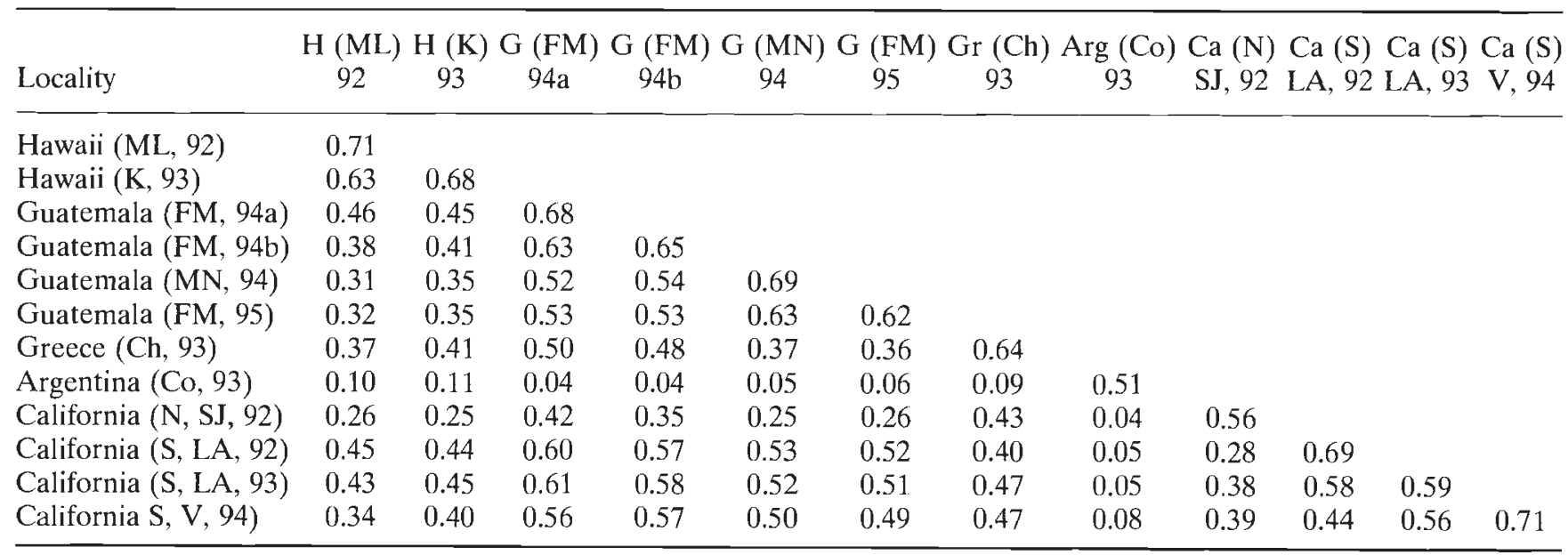

*The abbreviations used are: H (ML): Hawaii (M. Loa); H (K): Hawaii (Kauai); G (FM): Guatemala (Finca Mujulia); G (MN): Guatemala (Mundo Nuevo); Gr (Ch): Greece (Chios); Arg (Co): Argentina (Concordia); Ca (N, SJ): California (Northern, San Jose); Ca (S, LA): California (Southern, Los Angeles); Ca (S, V): California (Southern, Ventura).

lower compared with the average within-subpopulation values and they vary over a greater range $\left(s^{2}=0.013\right)$. A comparison of the betweensubpopulation similarity values in California and Guatemala shows that the means are significantly different ( $t$-test, $P<0.05)$, whereas the within-population values means are not ( $t$-test, $P>0.5$ ).

The frequency of each band morph was also determined based on presence/absence within each population. These data were converted to a distance measure (Nei, 1975) and used as the input matrix for another neighbour-joining tree (Fig. 5). This

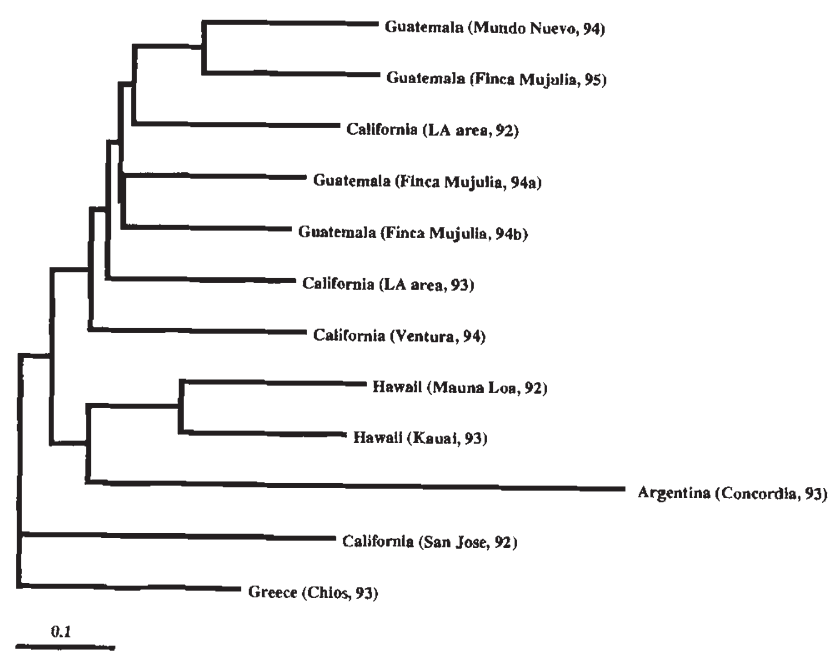

Fig. 3 Neighbour-joining tree for all medfly populations analysed here using distance relationships based on the similarity index. neighbour-joining tree was subjected to numerical resampling by bootstrapping (Felsenstein, 1988), and the resultant bootstrap values are shown at the tree branch points. Each value represents the number of times (out of 100 replications) that the represented groupings occurred in the resamplings. The bootstrap value of 99 shown at the base of this figure represents the support for a three-way branching at this point in the tree. Overall, the clustering in this tree is consistent with the first neighbour-joining tree. Bootstrapping was not possible with the first tree, because each distance is based on a single input value.

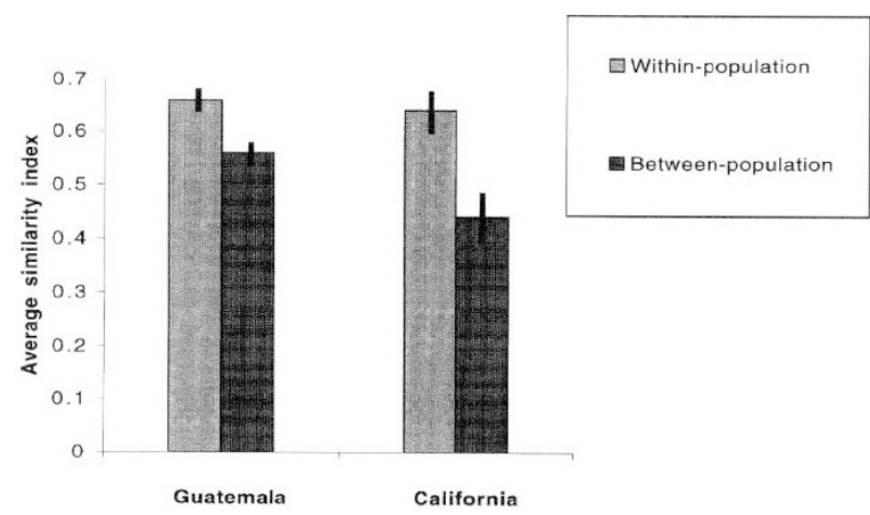

Fig. 4 Average within-population and between-population similarity indices for a series of Guatemalan medfly subpopulations compared with the various Californian samples analysed here. Standard errors of the means are indicated by a line within each bar.

(C) The Genetical Society of Great Britain, Heredity, 79, 302-309. 


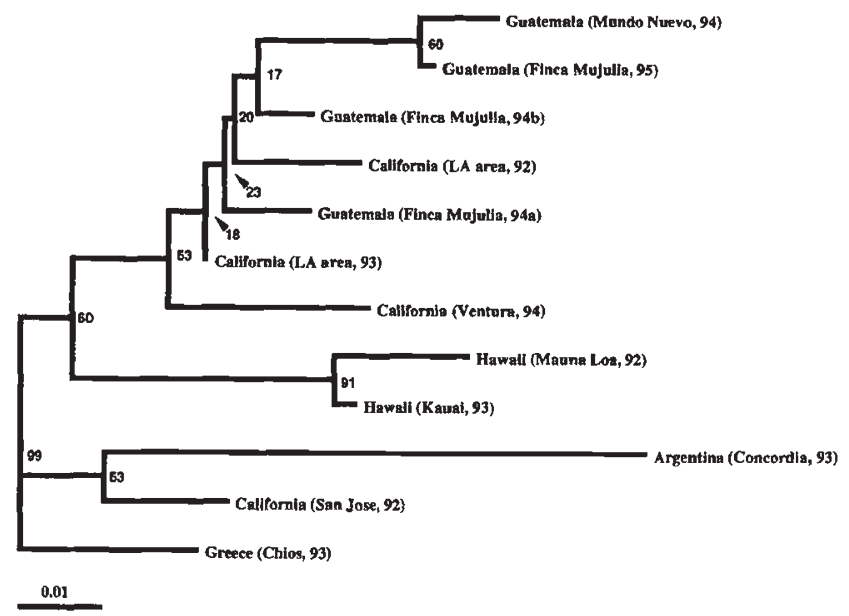

Fig. 5 Neighbour-joining tree for medfly populations based on distances derived from frequency data. Numbers at branch points are bootstrap values representing the number of times the branching was supported out of 100 replications.

\section{Discussion}

These results show that markers produced by arbitrarily primed PCR (referred to as RAPDs) can be used to document genetic relationships within and between populations of the medfly, including populations, such as those in California, which may represent the vanguard of attempts to invade and colonize a new habitat. These arbitrary priming methods have the advantage of identifying large amounts of genetic variation, but they do have some inherent limitations in terms of the analysis of the results obtained. With RAPDs, it is difficult to estimate heterozygosity or other classical population genetic parameters (Black, 1993; Slade et al., 1993) unless appropriate crosses can be performed to establish allelic relationships. We have documented Mendelian inheritance for many RAPD markers in our own work (He \& Haymer, unpublished observations), although it was not possible to conduct crosses using some of the samples listed here because only dead specimens were available (see Materials and methods). In many cases, RAPDs have been used to establish linkage relationships (see Yu \& Pauls, 1994), and this ability is clearly dependent on markers that are inherited in a Mendelian manner. In addition, there are some implicit assumptions about relationships of co-migrating bands in RAPD analyses, but this is generally considered to be a limitation only at levels above the types of intraspecific comparisons conducted here (Black, 1993).
We used RAPDs to document population relationships in a number of ways. One method included the calculation of a genetic similarity index based on the degree of band sharing between individuals. Using this method, one value obtained is a withinpopulation index measuring the genetic similarity among individuals within a sample. The withinpopulation similarity index values obtained here are all greater than 0.5 (maximum is 1.0 ), and this is consistent with the fact that individuals from the same population should represent a somewhat narrowly defined genetic pool. The same index is also used to calculate genetic similarity between populations. In this case, the index values vary over a much broader range. At one extreme are comparisons of subpopulations sampled within broadly defined geographical regions, such as Hawaii or Guatemala, which have established populations of the medfly. In these cases, the between-subpopulation comparison indices are again all greater than 0.5 , and in many cases they are nearly as high as the within-population values. This indicates that these subpopulations are genetically very similar to each other, probably as a result of substantial amounts of gene flow occurring between these populations. Gasperi et al. (1991) previously used allozyme studies to show the effect of gene flow in minimizing genetic distances between medfly populations. The high similarity values seen here are effectively equivalent to low genetic distance measures.

The first neighbour-joining tree constructed (Fig. 3) based on these values also shows that subpopulations from the same localities tend to cluster strongly together, again reflecting the minimal distances between these samples relative to the others. Neighbour-joining trees are constructed by creating nodes that link the least distant pairs of taxa (Saitou \& Nei, 1987). At the other extreme are populations, such as those in Greece and Argentina, which appear to be isolated gene pools because they exhibit (at least relatively) so little similarity to any of the other populations sampled here. Here again, these relationships can be seen both in the similarity indices and in the neighbour-joining tree. The second neighbour-joining tree, constructed using band frequencies (Fig. 5), also shows similar clustering relationships.

The ability of the similarity index to document genetic relationships between populations can be used to identify the possible origins of the medfly infestations that occurred in California over the period of 1992-94. Regardless of whether the Californian infestations reflect multiple, independent infestations or new outbreaks of a widespread estab- 
lished population, there should be a genetic relationship between infestations and the population that was the ultimate source of the invasion. Our results clearly indicate that, of the populations sampled here, at least some of the southern Californian infestations are most closely related to the Guatemalan populations. This is based on the fact that many of the between-population comparisons for Guatemala and southern California yield similarity indices greater than 0.5 , whereas other betweenpopulation comparisons do not. Both neighbourjoining trees also place the southern Californian samples in clusters with the Guatemalan populations, although to varying degrees. Although the statistical significance of such trees can be difficult to assess (Felsenstein, 1988), there are important features suggested by the relationships depicted here. Considering the second tree, extremely low bootstrap values are found for the clusters, including two of the southern Californian populations (92 and 93) and the Guatemalan populations. This is consistent with the idea that these populations are genetically so similar that they cannot be readily separated.

However, in terms of the actual origin of the southern Californian flies, it is important to note that the Guatemalan samples used here are probably convenient representatives of a more widespread Central American pattern of genetic markers. There is likely to be considerable homogenization through gene flow between a number of populations from this region, and it is possible that the Californian flies will exhibit similar or even closer relationships to flies from other countries, such as Honduras, Nicaragua or Costa Rica, all of which also harbour the medfly. These results, however, do clearly suggest that Hawaii is not a likely source of the flies constituting the 1992-94 infestations. An analysis of mitochondrial DNA by Sheppard et al. (1992) on medflies found in California in 1989 and 1991 also concluded that Hawaii was not a likely source of the infestation.

In terms of the relationships of the different Californian infestations to each other, they appear to be different in several respects in comparison with the other subpopulation samplings described above. Among the Californian samples, most of the between-subpopulation comparisons produce similarity indices that fall below 0.5 . In one case (southern California, LA area, 1992 compared with northern California, San Jose, 1992), the value is 0.28 , one of the lowest between-population values obtained in this study. Overall, the average of the between-population values for the Californian infest- ations is statistically significantly different compared with a similar set of between-population values for Guatemala. These results immediately suggest that the different Californian infestations are not as related to each other as are the Guatemalan subpopulations. If the different infestations in California represented multiple samplings of the same population, the level of similarity between these populations should be approximately the same, because the Guatemalan subpopulations are defined in similar temporal and spatial terms.

The neighbour-joining trees also depict some interesting relationships involving the different Californian infestations. The northern California 92 infestation appears to be completely unrelated to any of the southern California infestations because of its location on a separate branch of both trees. In the bootstrap analysis conducted using the tree based on frequency data, this is the most wellsupported separation in the data set. Although the separations involving the southern California 92 and 93 samples are not well supported (as described above), the separation of the Californian flies from Ventura (1994) from the other Californian infestations is much more strongly supported. Based on the notion that legitimate groupings should occur in at least half of the bootstrap estimates (Felsenstein, 1988), this infestation can also be considered to be separate from the others. In addition, closer examination of the patterns produced by individual primers (such as X16) show the presence of unique or private alleles in flies representing the 1994 infestation compared with any of the other Californian infestations.

Overall, these results show that the various Californian infestations (from 1992 to 1994) exhibit genetic differences, especially compared with a set of similar population samples from Guatemala. The genetic make-up of the flies from northern California in 1992, in particular, is clearly different from any of the southern Californian infestations. Even within southern California, the different infestations exhibit less similarity to each other than do similar subpopulations from Guatemala and Hawaii. The observation of unique alleles in the 1994 infestation in southern California (Ventura, 1994) and other genetic differences argues against the possibility of these flies being a subset of earlier infestations. The 1992 and 1993 infestations in southern California are very closely related to each other, but at this time it is not possible to determine whether this close relationship is caused by the resurgence of a population that was suppressed but not eradicated or as a result of similar origins of independent 
infestations. In terms of the original questions raised by Carey (1991) regarding the status of the medfly in California, overall our results argue against the hypothesis of a single, established population distributed throughout the entire state and support the hypothesis of multiple introductions having occurred in this timeframe. Our results also indicate that, of the populations considered here, at least for the years 1992 and 1993, Central America is a likely source of the flies that invaded the southern California region.

\section{Acknowledgements}

This work was supported by grant 95-81000214(GR) from the USDA (APHIS) and a grant from the Citrus Research Board of California. The authors also wish to acknowledge the support of the Medfly Germplasm Repository and the CDFA in providing samples, and the help of two anonymous reviewers in suggesting improvements to the manuscript.

\section{References}

BARUfFI，L., DAMIANI，G., GUGLIEMO, C. R., BANDI， C., MAlACRIDA, A. R. AND GASPERI, G. 1995. Polymorphism within and between populations of Ceratitis capitata: comparison between RAPD and multilocus enzyme electrophoresis data. Heredity, 74, 425-437.

BLACK, W. C., IV. 1993. PCR with arbitrary primers: approach with care. Insect Mol. Biol., 2, 1-6.

CAREY, J. 1991. Establishment of the Mediterranean fruit fly in California. Science, 253, 1369-1373.

FELSENSTEIN, J. 1988. Phylogenies from molecular sequences: inference and reliability. Ann. Rev. Genet., 22, 521-565.

FIMIANI, P. 1989. The Mediterranean region. In: Robinson, A. S. and Hooper, G. (eds) Fruit Flies: their Biology, Natural Enemies and Control, pp. 37-50. Elsevier, New York.

FISCHER-COLBRIE, P. AND BUSCH-PETERSEN, E. 1989. Temperate Europe and West Asia. In: Robinson, A. S. and Hooper, G. (eds) Fruit Flies: their Biology, Natural Enemies and Control, pp. 91-100. Elsevier, New York.

GASPERI, G., GUGLIELMINO, C. R., MALACRIDA, A. R. AND MILANI, R. 1991. Genetic variability and gene flow in geographical populations of Ceratitis capitata. Heredity, 67, 347-356.

HAYMER, D. S. 1994. Random amplified polymorphic DNAs and microsatellites. What are they and can they tell us anything we don't already know? Ann. Entomol. Soc. Am., 87, 717-722.
HAYMER, D. S. AND McINNIS, D. 1992. Resolution of populations of the Mediterranean fruit fly at the DNA level using random primers for the polymerase chain reaction. Genome, 37, 244-248.

KAMBHAMPATI, S., BLACK, W. C., IV AND RAI, K. 1991. Random amplified polymorphic DNA of mosquito species and populations: techniques, statistical analysis and applications. J. Med. Entomol., 29, 939-945.

MALACRIDA, A. R., GUGLIELMINO, C. R., GASPERI, G., BARUFFI, L. AND MILANI, R. 1992. Spatial and temporal differentiation in colonizing populations of Ceratitis capitata. Heredity, 69, 101-111.

MUllis, K., FALCOMA, F., SCHARF, S., SNIKL, R., HORN, G. AND EHRLICH, H. 1986. Specific amplification of DNA in vitro: the polymerase chain reaction. Cold Spring Harb. Symp. Quant. Biol., 51, 260.

NEI, M. 1975. Molecular Population Genetics and Evolution. Elsevier, New York.

NEI, M. AND L1, w.-H. 1979. Mathematical models for studying genetic variation in terms of restriction endonucleases. Proc. Natl. Acad. Sci. U.S.A., 76, 5269-5273.

SAITOU, N. AND NEI, M. 1987. The neighbor-joining method: a new method for constructing phylogenetic trees. Mol. Biol. Evol., 4, 406-425.

SAul, s. 1986. Genetics of the Mediterranean fruit fly, Ceratitis capitata. Agric. Zool. Rev., 1, 73-108.

SHEPPARD, w. S., STECK, G. J. AND McPHERON, B. A. 1992. Geographic populations of the medfly may be differentiated by mitochondrial DNA variation. Experientia, 48, 1010-1013.

SLADE, R. W., MORITZ, C., HEIDEMAN, A. AND HALE, P. T. 1993. Rapid assessment of single-copy nuclear DNA variation in diverse species. Mol. Ecol., 2, 359-373.

SWOFFORD, D. L. AND OLSEN, G. J. 1990. Phylogeny reconstruction. In: Hillis, D. and Moritz, C. (eds) Molecular Systematics, pp. 411-500. Sinauer, Sunderland, MA.

WELSH, J. AND MCCLELLAND, M. 1990. Fingerprinting genomes using PCR with arbitrary primers. Nucl. Acids Res., 18, 7213-7218.

WELSH, J., RALPH, D. AND MCCLELLAND, M. 1995. DNA and RNA fingerprinting using arbitrarily primed PCR. In: Innis, M., Gelfand, D. and Sninsky, J. (eds) PCR Strategies, pp. 249-276. Academic Press, New York.

WILliAMS, J. G. K., KUBELIK, A., LIVAK, K., RAFALSKI, J. AND TINGEY, s. 1990. DNA polymorphisms amplified by arbitrary primers are useful as genetic markers. Nucl. Acids Res., 18, 6531-6535.

WILliams, C. L., GOldSON, S., BAIRD, D. AND BUllock, D. 1994. Geographical origin of an introduced insect pest, Listronotus bonariensis, determined by RAPD analysis. Heredity, 72, 412-419.

YU, K. AND PAULS, K. 1994. The use of RAPD analysis to tag genes and determine relatedness in heterogeneous plant populations using tetraploid alfalfa as an example. In: Griffin, H. and Griffin, A. (eds) PCR Technology, pp. 201-214. CRC Press, Boca Raton, FL. 